\title{
Projectile excitation in strongly asymmetric ion-atom collisions
}

\author{
U Thumm, J S Briggs and O Schöller \\ Fakultät für Physik, Universität Freiburg, D.78 Freiburg, West Germany
}

Received 22 September 1987

\begin{abstract}
In an ion-atom collision at velocity $v$ such that $v \gg Z_{\mathrm{T}}$, the nuclear charge of the target, the first Born approximation is expected to be valid. However, if at the same time $Z_{\mathrm{P}} \gg Z_{\mathrm{T}}$, where $Z_{\mathrm{P}}$ is the projectile nuclear charge, the first Born approximation is never valid at non-relativistic velocities since the projectile field cannot be regarded as a perturbation. A theory of inelastic processes in such ion-atom collisions is presented and applied specifically to the case of excitation of $\mathrm{K}$-shell projectile electrons by the initially neutral target atom. The theory is formulated in such a way as to emphasise the polarisation of the target by the strong projectile ionic field and to see immediately the departures from the first Born approximation. The principal effects are that the target nucleus and electrons act coherently in exciting the projectile, rather than incoherently as in the first Born approximation, and that collisions in which the target remains in its ground state are greatly suppressed compared with the first Born result.
\end{abstract}

\section{Introduction}

The theory of the collision between partially stripped ions and target atoms is undergoing rapid development under the impetus of new experimental results made possible by technological advances in ion sources and accelerating techniques. Particularly noteworthy and of direct interest here is the use of few-electron highly charged ions as projectiles, in which the processes of projectile excitation, projectile ionisation, electron capture to the projectile continuum and simultaneous electron excitation and capture are readily observed. The theoretical description of such collision processes requires an extension of existing theories to incorporate the effect of the strong field of the incident ion and the dynamical role played by the electron-electron interactions. The two aspects are quite separate of course and the problem of the dynamical treatment of projectile-electron-target-electron interaction persists when projectiles of low charge are used.

Any theory of few-body collisions seeks to identify simplifications permissible under certain limiting values of the dynamical parameters. For example, when the collision velocity is low compared with electronic orbital velocities the molecular or adiabatic description of a collision is used. Here the electron-nucleus and electron-electron interactions can be calculated to a high degree of accuracy for arbitrary nuclear charges at each fixed internuclear distance, with the nuclear motion appearing as the perturbing element (Briggs 1976). At higher collision velocities the adiabatic assumption, particularly as regards the electron-electron interaction, breaks down and alternative 
approaches involving atomic basis set expansions or explicit high-order many-particle scattering theory, as considered in this work, are necessary.

In the limit of collision velocity much greater than the electron orbital velocities, the first Born approximation is appropriate when the effective electron-nuclear forces are of the same order of magnitude as the electron-electron force. The exchange interaction between projectile and target electrons may be neglected and the transition amplitude factors into a part dependent only upon projectile properties and a part dependent upon target properties. The collision is characterised by an impulsive exchange of momentum between the two systems, as is always the case in the first Born approximation. The first Born theory was originally developed for collisions between one-electron atoms and ions (Bates and Griffing 1953, 1954, 1955) but the extension to many-electron systems is straightforward (Briggs and Taulbjerg 1978, Gillespie et al 1978, Hartley and Walters 1987).

The first Born theory applies under the condition that the velocity $v$ is such that the perturbing part of the electron-nucleus interaction, whose strength is characterised by the Sommerfeld parameter $\nu=Z / v$, where $Z$ is the effective charge from either nucleus, is small, i.e. $\nu \ll 1$. When this is true, the electron-electron perturbation of relative strength $1 / Z$ is even smaller. The non-relativistic theory requires further that $v \ll c$, the speed of light. The essential point to be emphasised in this paper is that current experiments use projectiles of such high charge $Z$ that $Z / v \ll 1$ cannot be reached before the condition $v \ll c$ is violated, that is the non-relativistic first Born approximation is never applicable to these collisions, even though the collision velocity may be large compared with target electron orbital velocities. The first Born theory assumes that the undistorted target wavefunction may be used in the transition matrix element. However when $Z_{\mathrm{P}} / v$ is not small (where $Z_{\mathrm{P}}$ is the projectile effective charge) it is clear that severe distortion of the target wavefunction may accompany any inelastic scattering event. The object of this paper is to develop a theory of collisions between few-electron highly charged projectile ions and light neutral targets in which the distortion of the target is explicitly taken into account. The particular process to be considered will be excitation of a single projectile electron where the final state of the target is not monitored. The approach used is similar to that employed in the development of the impulse approximation (McDowell 1961, Coleman 1969) or the strongpotential Born approximation (Macek and Alston 1982) for charge exchange in ionatom collisions. The similarity lies essentially in the approximation of the full Green operator, involving both nucleus-electron potentials, by one involving only one of the nucleus-electron potentials.

It should be noted that a theory of projectile excitation using the Schwinger variational principle in a time-dependent formalism has been developed by Brendle et al (1985). For collisions with $Z_{\mathrm{P}} \gg Z_{\mathrm{T}}$ they find that higher-order scattering processes are important. Our theory, although derived from a different point of view, leads to the same qualitative conclusion.

The theory will be developed as follows. In $\S 2$ the formal scattering theory for an ion-atom collision leading to excitation of the projectile and/or target will be developed for the simplified case of a one-electron projectile and target. In the situation where the projectile residual charge is much greater than the target nuclear charge a form of the strong-potential Born approximation (Macek and Alston 1982) is developed in which the polarisation of the target by the projectile is represented explicitly. The resulting $T$-matrix element for projectile excitation is evaluated approximately in $\S 3$ where the ensuing differences from results obtained within the first Born approximation 
are particularly emphasised. In $\S 4$ numerical results for total cross sections for projectile excitation by helium atoms are presented for incident ions of increasing nuclear charge. Atomic units are used throughout.

\section{The $T$ matrix for projectile excitation}

The chosen coordinates in which to describe the collision of a one-electron ion with a target one-electron ion or atom under the condition $Z_{\mathrm{P}} \gg Z_{\mathrm{T}}$ are shown in figure 1 , where $\mathrm{P}, \mathrm{T}, \mathrm{P}^{\prime}$ are the centre of mass of projectile, target and projectile together with the target electron, respectively. The vector $\boldsymbol{r}_{\mathrm{P}}^{\prime}$ gives the position of the target electron with respect to $\mathrm{P}^{\prime}$. The projectile and target masses are $M_{\mathrm{P}}$ and $M_{\mathrm{T}}$ respectively and the electron mass $m$ is numerically equal to unity. Definition of the mass ratios

$$
\alpha=\frac{M_{\mathrm{T}}}{M_{\mathrm{T}}+m} \quad \beta=\frac{M_{\mathrm{P}}}{M_{\mathrm{P}}+m} \quad \beta^{\prime}=\frac{M_{\mathrm{P}}+m}{M_{\mathrm{P}}+2 m}
$$

allows the transformation between the coordinates to be expressed as

$$
\boldsymbol{r}_{\mathrm{P}}^{\prime}=\alpha \boldsymbol{r}_{\mathrm{T}}-\boldsymbol{R}_{\mathrm{T}} \quad \boldsymbol{R}_{\mathrm{P}}=\left(\alpha \beta^{\prime}-1\right) \boldsymbol{r}_{\mathrm{T}}-\beta^{\prime} \boldsymbol{R}_{\mathrm{T}}
$$

and

$$
\boldsymbol{r}_{\mathrm{T}}=\beta^{\prime} \boldsymbol{r}_{\mathrm{P}}^{\prime}-\boldsymbol{R}_{\mathrm{P}} \quad \boldsymbol{R}_{\mathrm{T}}=\left(\alpha \beta^{\prime}-1\right) \boldsymbol{r}_{\mathrm{P}}^{\prime}-\alpha \boldsymbol{R}_{\mathrm{P}}
$$

The various interparticle vectors appearing in the Coulomb interactions involved are, apart from $\boldsymbol{r}_{\mathrm{P}}$ and $\boldsymbol{r}_{\mathrm{T}}$,

$$
\begin{aligned}
& \boldsymbol{\rho}=(\beta-1) \boldsymbol{r}_{\mathrm{P}}+\left(\beta^{\prime}-1\right) \boldsymbol{r}_{\mathrm{P}}^{\prime}-\boldsymbol{R}_{\mathrm{P}} \\
& \boldsymbol{r}_{\mathrm{T}}-\rho=(1-\beta) \boldsymbol{r}_{\mathrm{P}}+\boldsymbol{r}_{\mathrm{P}}^{\prime} \\
& \boldsymbol{r}_{\mathrm{P}}+\boldsymbol{\rho}=\beta \boldsymbol{r}_{\mathrm{P}}+\left(\beta^{\prime}-1\right) \boldsymbol{r}_{\mathrm{P}}^{\prime}-\boldsymbol{R}_{\mathrm{P}} \\
& \boldsymbol{r}_{\mathrm{P}}+\boldsymbol{\rho}-\boldsymbol{r}_{\mathrm{T}}=\beta \boldsymbol{r}_{\mathrm{P}}-\boldsymbol{r}_{\mathrm{P}}^{\prime} .
\end{aligned}
$$

In defining the $T$-matrix element for excitation of the projectile the initial and final states must be specified. In the initial state the two electrons are infinitely separated and the initial state reads

$$
\left\langle\boldsymbol{R}_{\mathrm{T}}, \boldsymbol{r}_{\mathrm{P}}, \boldsymbol{r}_{\mathrm{T}} \mid \Phi_{i}\right\rangle=(2 \pi)^{-3 / 2} \exp \left(\mathrm{i} \boldsymbol{K}_{i} \cdot \boldsymbol{R}_{\mathrm{T}}\right) \varphi_{i}^{\mathrm{P}}\left(\boldsymbol{r}_{\mathrm{P}}\right) \varphi_{i}^{\mathrm{T}}\left(\boldsymbol{r}_{\mathrm{T}}\right)
$$

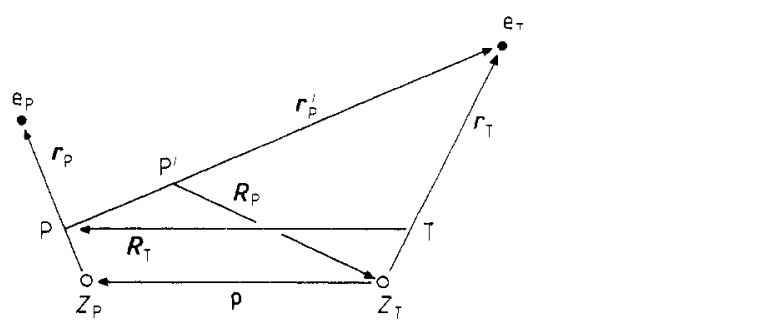

Figure 1. Coordinates for $\mathrm{H}$-like collision partners. 
where $\varphi_{i}^{p}$ is the initial bound state of the projectile electron and $\varphi_{i}^{T}$ that of the target electron. The relative momentum $\boldsymbol{K}_{i}$ of projectile ion and target atom is

$$
\boldsymbol{K}_{i}=\frac{\left(M_{\mathrm{T}}+m\right)\left(M_{\mathrm{P}}+m\right)}{M_{\mathrm{T}}+M_{\mathrm{P}}+2 m} \boldsymbol{v}_{i}
$$

where $v_{i}$ is the initial relative velocity of the colliding partners.

The final state is more difficult. Comparison will be made with experiments in which the final state of the target electron is not monitored. Hence a summation will be made over a complete set of the unobserved target electron states. Nevertheless these states include excited and continuum states of both target and projectile as final physical states. Here, to carry out this summation, the final relative momentum of the colliding partners will be taken to be that calculated with the initial target electron referred to the target nucleus. Then the final state of the relative motion is written

$$
\left\langle\boldsymbol{R}_{\mathrm{T}}, \boldsymbol{r}_{\mathrm{P}}, \boldsymbol{r}_{\mathrm{T}} \mid \Phi_{f}\right\rangle=(2 \pi)^{-2 / 3} \exp \left(\mathrm{i} \boldsymbol{K}_{f} \cdot \boldsymbol{R}_{\mathrm{T}}\right) \varphi_{f}^{\mathrm{P}}\left(\boldsymbol{r}_{\mathrm{P}}\right) \varphi_{f}^{\mathrm{T}}\left(\boldsymbol{r}_{\mathrm{T}}\right)
$$

where $\varphi_{f}^{\mathrm{P}}$ is the final projectile excited state and $\varphi_{f}^{\mathrm{T}}$ is the unspecified final state of the target electron over which a summation will be made. The relative momentum of the final products is written as

$$
\boldsymbol{K}_{f}=\frac{\left(M_{\mathrm{T}}+m\right)\left(M_{\mathrm{P}}+m\right)}{M_{\mathrm{T}}+M_{\mathrm{P}}+2 m} \boldsymbol{v}_{f}
$$

where $v_{f}$ is the final relative velocity. Strictly speaking, for those final states for which the unobserved target electron is bound to the projectile one should define

$$
\boldsymbol{K}_{f}=\frac{M_{\mathrm{T}}\left(M_{\mathrm{P}}+2 m\right)}{M_{\mathrm{T}}+M_{\mathrm{P}}+2 m} \boldsymbol{v}_{f}
$$

but this only introduces errors in the definition of $\boldsymbol{K}_{f}$ which are of order $\left(m / M_{\mathrm{p}}\right)$.

In writing down the final state (2.7), the exchange of electrons has been neglected, i.e. the final two-electron state is not antisymmetrised. Although formally easy to include, exchange is omitted here to considerably simplify the nevertheless formidable subsequent calculation of the $T$-matrix elements. Its omission is justified on the grounds that the two electrons mostly occupy two very different parts of space during the collision. The projectile electron is involved in a transition from its tightly bound $1 \mathrm{~s}$ orbital into the lowest lying excited states. As we will require the condition $Z_{\mathrm{T}} \ll Z_{\mathrm{P}}$ for the validity of subsequent approximations, the target electron begins in an orbit rather diffuse compared with the projectile electron orbits and most of the weight in the summation over unobserved states will come from such diffuse states.

With this definition of initial and final states the $T$-matrix element for projectile excitation reads

$$
T_{f i}=\left\langle\Phi_{f}|T| \Phi_{i}\right\rangle=\left\langle\Phi_{f}\left|V_{i}\left(1+G^{+} V_{i}\right)\right| \Phi_{i}\right\rangle
$$

where $G^{+}(E)=(E-H+\mathrm{i} \delta)^{-1}$ and $H$ is the full Hamiltonian of the four interacting particles with total energy $E$. The perturbing potential $V_{i}$ is that part of the full Coulomb interaction which is not diagonalised in the initial and final states, i.e.

$$
V_{i}=H-H_{i}
$$

where

$$
H_{i}=K\left(\boldsymbol{R}_{\mathrm{T}}, r_{\mathrm{P}}, r_{\mathrm{T}}\right)+V_{\mathrm{Pp}}\left(r_{\mathrm{P}}\right)+V_{\mathrm{T}}\left(r_{\mathrm{T}}\right)
$$


Here $K$ is the total kinetic energy of the system of two electrons and two nuclei, $V_{\mathrm{Pp}}$ is the Coulomb interaction between projectile nucleus $\mathrm{P}$ and projectile electron $\mathrm{p}$ and $V_{\mathrm{T}}$ that between target nucleus $\mathrm{T}$ and target electron $\mathrm{t}$. The residual interaction $V_{\mathrm{i}}$ is then composed of four elements, i.e.

$$
V_{i}=V_{\mathrm{Pt}}+V_{\mathrm{Tp}}+V_{\mathrm{pt}}+V_{\mathrm{TP}}
$$

In the following, the internuclear repulsion $V_{\mathrm{TP}}$ will be consistently neglected, i.e. rectilinear trajectories will be assumed for initial and final relative motion of the collision fragments. At the velocities considered here the trajectories contributing most to the total cross section correspond to only small-angle deflection.

The $T$-matrix element $(2.10)$ can be written

$$
T_{f i}=\left\langle\Phi_{f}\left|V_{i}\right| \Psi_{i}^{\top}\right\rangle
$$

where $\Psi_{i}^{+}$is the exact scattering state

$$
\left|\Psi_{i}^{+}\right\rangle=\left(1+G^{+} V_{i}\right)\left|\Phi_{i}\right\rangle \text {. }
$$

Here $\Psi_{i}^{+}$will be approximated by simplifying the potential $V_{i}$ occurring both explicitly and in the Green operator $G^{+\cdot}=\left(E-H_{0}-V_{\mathrm{i}}+i \delta\right)^{-1}$ in (2.15). This simplification will be appropriate to the situation $Z_{\mathrm{P}} \gg Z_{\mathrm{T}}$.

Since the major new effect to be incorporated in the theory here is the polarisation of the target ground state, mostly occurring at internuclear distances larger than the projectile ground-state orbital radius, the combined effect of the projectile ion on the target electron will be incorporated into a single effective ionic potential, i.e.

$$
\begin{aligned}
\tilde{V}_{\mathrm{Pt}} & =V_{\mathrm{Pt}}+V_{\mathrm{pt}} \\
& =-\frac{Z_{\mathrm{P}}}{\left|\boldsymbol{r}_{\mathrm{T}}-\boldsymbol{\rho}\right|}+\frac{1}{\left|\boldsymbol{\rho}+\boldsymbol{r}_{\mathrm{P}}-\boldsymbol{r}_{\mathrm{T}}\right|} \\
& \simeq-\frac{\left(Z_{\mathrm{p}}-1\right)}{r_{\mathrm{P}}^{\prime}} \quad \text { for } \rho \gg r_{\mathrm{P}} .
\end{aligned}
$$

The total Hamiltonian can now be divided in two ways, one in which the target electron feels the effect of the projectile ion potential $\tilde{V}_{\mathrm{Pt}}$ and one in which it feels predominantly the target nuclear potential $V_{\mathrm{Tt}}$, i.e.

or

$$
\begin{aligned}
H & =\left(K+V_{\mathrm{Pp}}+\tilde{V}_{\mathrm{Pt}}\right)+V_{\mathrm{Tt}}+V_{\mathrm{Tp}} \\
& =H_{\mathrm{P}}+V_{\mathrm{Tt}}+V_{\mathrm{Tp}}
\end{aligned}
$$

$$
\begin{aligned}
H & =\left(K+V_{\mathrm{Pp}}+V_{\mathrm{Tt}}\right)+\tilde{V}_{\mathrm{Pt}}+V_{\mathrm{Tp}} \\
& =H_{\mathrm{T}}+\tilde{V}_{\mathrm{Pt}}+V_{\mathrm{Tp}} .
\end{aligned}
$$

At this stage a strong-potential approximation will be invoked in assuming that the projectile electron, to be excited, propagates predominantly during the collision under the influence of the projectile potential $V_{\mathrm{Pp}}$. Then $V_{\mathrm{Tp}}$ can be dropped from $(2.15)$, (2.17) and (2.18). It will still be retained in the transition matrix element (2.14), of course, amounting to a first-order strong-potential Born (SPB) approximation in the potentials $V_{\mathrm{Pp}}$ and $V_{\mathrm{Tp}}$. Now the exact scattering state $(2.15)$ is approximated by

$$
\left|\Psi_{i}^{+(\mathrm{SPB})}\right\rangle=\left(1+G_{\mathrm{P}}^{+} \tilde{V}_{\mathrm{Pt}}\right)\left|\Phi_{i}\right\rangle
$$


where

$$
G_{\mathrm{P}}^{\top}=\left[E-H_{\mathrm{P}}+\mathrm{i} \delta\right]^{-1} \text {. }
$$

One sees that this strong-potential approximation considers the major distortion of the target initial state to be represented by virtual states of the projectile, i.e. by exchange polarisation. The direct polarisation, represented by virtual excitation into target excited states is considered to be less important in the situation in which $Z_{\mathrm{P}} \gg Z_{\mathrm{T}}$ and is neglected.

\section{Evaluation of the $T$-matrix element}

We decompose the motion of the target nucleus and the target electron in plane waves with momenta $\boldsymbol{k}_{\mathrm{T}}$ and $\boldsymbol{k}$ with respect to the centres of mass $\mathrm{P}$ of the projectile nucleus and electron and $\mathrm{P}^{\prime}$ of the projectile nucleus, the projectile electron and the target electron respectively (compare figure 1 ). With

$\left|\Phi_{i}\right\rangle=\left|\boldsymbol{K}_{i}, \varphi_{i}^{\mathrm{P}}, \varphi_{i}^{\mathrm{T}}\right\rangle \quad\left\langle\boldsymbol{r}_{\mathrm{P}}^{\prime}, \boldsymbol{R}_{\mathrm{P}} \mid \boldsymbol{k}, \boldsymbol{k}_{\mathrm{T}}\right\rangle=(2 \pi)^{-3} \exp \left(\mathrm{i} \boldsymbol{k} \cdot \boldsymbol{r}_{\mathrm{P}}^{\prime}\right) \exp \left(\mathrm{i} \boldsymbol{k}_{\mathrm{T}} \cdot \boldsymbol{R}_{\mathrm{P}}\right)$

we find

$$
\left|\Psi_{i}^{+(\mathrm{SPB})}\right\rangle=\left|\varphi_{i}^{\mathrm{P}}\right\rangle \int d \boldsymbol{k}_{\mathrm{T}} \mathrm{d} \boldsymbol{k}\left(1+G_{\mathrm{P}}^{+} \tilde{V}_{\mathrm{Pt}}\right)\left|\boldsymbol{k}, \boldsymbol{k}_{\mathrm{T}}\right\rangle\left\langle\boldsymbol{k}, \boldsymbol{k}_{\mathrm{T}} \mid \boldsymbol{K}_{i}, \varphi_{i}^{\mathrm{T}}\right\rangle
$$

where the overlap integral is given by

$$
\begin{aligned}
\left\langle\boldsymbol{k}, \boldsymbol{k}_{\mathrm{T}} \mid \boldsymbol{K}_{i}, \varphi_{i}^{\mathrm{T}}\right\rangle & =(2 \pi)^{-9 / 2} \int \mathrm{d} \boldsymbol{r}_{\mathrm{T}} \mathrm{d} \boldsymbol{R}_{\mathrm{T}} \exp \left(-\mathrm{i} \boldsymbol{k} \cdot \boldsymbol{r}_{\mathrm{P}}^{\prime}-\mathrm{i} \boldsymbol{k}_{\mathrm{T}} \cdot \boldsymbol{R}_{\mathrm{P}}+\mathrm{i} \boldsymbol{K}_{i} \cdot \boldsymbol{R}_{\mathrm{T}}\right) \boldsymbol{\varphi}_{i}^{\mathrm{T}}\left(\boldsymbol{r}_{\mathrm{T}}\right) \\
& =\delta\left(\boldsymbol{k}+\boldsymbol{\beta}^{\prime} \boldsymbol{k}_{\mathrm{T}}+\boldsymbol{K}_{i}\right) \tilde{\varphi}_{i}^{\mathrm{T}}\left[\alpha \boldsymbol{k}-\left(1-\alpha \beta^{\prime}\right) \boldsymbol{k}_{\mathrm{T}}\right]
\end{aligned}
$$

and $\tilde{\varphi}_{i}^{\mathrm{T}}$ is the initial target state in momentum space. Fourier transforms are performed according to

$$
\tilde{f}(\boldsymbol{k})=(2 \pi)^{-3 / 2} \int \mathrm{d} \boldsymbol{r} f(\boldsymbol{r}) \exp (-\mathrm{i} \boldsymbol{k} \cdot \boldsymbol{r}) .
$$

Represented in the appropriate coordinates the remaining factor in (3.2) separates as $\left\langle\boldsymbol{r}_{\mathrm{P}}, \boldsymbol{r}_{\mathrm{P}}^{\prime}, \boldsymbol{R}_{\mathrm{P}} \mid \varphi_{i}^{\mathrm{P}}\right\rangle\left(1+G_{\mathrm{P}}^{+} \tilde{V}_{\mathrm{Pt}}\right)\left|\boldsymbol{k}, \boldsymbol{k}_{\mathrm{T}}\right\rangle=(2 \pi)^{-3 / 2} \varphi_{i}^{\mathrm{P}}\left(\boldsymbol{r}_{\mathrm{P}}\right) \exp \left(\mathrm{i} \boldsymbol{k}_{\mathrm{T}} \cdot \boldsymbol{R}_{\mathrm{P}}\right) \Psi_{\boldsymbol{k}, \varepsilon}^{+}\left(\boldsymbol{r}_{\mathrm{P}}^{\prime}\right)$

where the off-shell wavefunction $\Psi_{k, \varepsilon}^{+}$is defined by

$$
\begin{aligned}
& \Psi_{k, \varepsilon}^{+}\left(\boldsymbol{r}_{\mathrm{P}}^{\prime}\right)=(2 \pi)^{-3 / 2}\left(1+G_{\mathrm{P}}^{+}(\varepsilon) \tilde{V}_{\mathrm{Pt}}\right) \exp \left(\mathrm{i} \boldsymbol{k} \cdot \boldsymbol{r}_{\mathrm{P}}^{\prime}\right) \\
& G_{\mathrm{P}}^{+}(\varepsilon)=\left(\varepsilon-K\left(\boldsymbol{r}_{\mathrm{P}}^{\prime}\right)-\tilde{V}_{\mathrm{Pt}_{\mathrm{t}}}+\mathrm{i} \delta\right)^{-1} \\
& \varepsilon=E-\varepsilon_{i}^{\mathrm{P}}-\frac{1}{2 \mu} k_{\mathrm{T}}^{2}=\frac{1}{2 M} K_{i}^{2}-\frac{1}{2 \mu} k_{\mathrm{T}}^{2}+\varepsilon_{i}^{\mathrm{T}} .
\end{aligned}
$$

The second term in (3.6) takes account of the exchange polarisation. Omission of this term leads us back to the first Born approximation, so that replacement of $\Psi_{k, \varepsilon}^{+}$by its plane wave part provides an easy check on the SPB approximation. In $(3.7), K\left(\boldsymbol{r}_{\mathrm{P}}^{\prime}\right)$ is the kinetic energy operator of the target electron and $\varepsilon_{i}^{\mathrm{P}}, \varepsilon_{i}^{\top}$ the initial binding energies of projectile and target electron, respectively. Note that $M$ and $\mu$ denote two different reduced masses

$$
M=\frac{\left(M_{\mathrm{P}}+m\right)\left(M_{\mathrm{T}}+m\right)}{M_{\mathrm{P}}+M_{\mathrm{T}}+2 m} \quad \mu=\frac{\left(M_{\mathrm{P}}+2 m\right) M_{\mathrm{T}}}{M_{\mathrm{P}}+M_{\mathrm{T}}+2 m}
$$


according to whether the target electron is bound to the target or projectile nucleus, respectively. With (3.2), (3.3) and (3.5) the scattering wavefunction is given by

$$
\begin{aligned}
& \left\langle\boldsymbol{r}_{\mathrm{P}}, \boldsymbol{r}_{\mathrm{P}}^{\prime}, \boldsymbol{R}_{\mathrm{P}} \mid \Psi_{i}^{+(\mathrm{SPB})}\right\rangle \\
& =(2 \pi)^{-3 / 2} \beta^{\prime-1} \varphi_{i}^{\mathrm{P}}\left(\boldsymbol{r}_{\mathrm{P}}\right) \int \mathrm{d} \boldsymbol{k} \exp \left[-\beta^{-1}\left(\boldsymbol{k}+\boldsymbol{K}_{i}\right) \boldsymbol{R}_{\mathrm{P}}\right] \Psi_{k, \varepsilon}^{+}\left(\boldsymbol{r}_{\mathrm{P}}^{\prime}\right) \tilde{\varphi}_{i}^{\top}\left(\beta^{\prime-1} \boldsymbol{k}+\boldsymbol{v}\right) .
\end{aligned}
$$

This result can be interpreted as follows. The initial projectile state remains undistorted. The initial target state is decomposed in plane waves with momentum $\boldsymbol{k}$, which, under the influence of $\tilde{V}_{\mathrm{Pt}}$, are modified to intermediate distorted states described by $\Psi_{k, F}^{+}$. These wavefunctions are off shell, since in general $\frac{1}{2} k^{2} \neq \varepsilon$. Finally, the off-shell wavefunctions are averaged over the initial momentum distribution of the target electron. The plane wave factor in the integrand describes the rectilinear motion of the target nucleus with respect to $\mathrm{P}^{\prime}$.

We now use (3.10) to calculate the $T$-matrix element

$$
T_{i f}^{(\mathrm{SPB})}=\left\langle\Phi_{f}\left|V_{\mathrm{Tp}}+V_{\mathrm{tp}}+V_{\mathrm{Pt}}\right| \Psi_{i}^{+(\mathrm{SPB})}\right\rangle
$$

using the Fourier transforms

$$
\begin{aligned}
& V_{\mathrm{Tp}}=-\frac{1}{2 \pi^{2}} Z_{\mathrm{T}} \int \frac{\mathrm{d} \boldsymbol{q}}{q^{2}} \exp \left\{\mathrm{i}\left[\beta \boldsymbol{r}_{\mathrm{P}}-\left(1-\beta^{\prime}\right) \boldsymbol{r}_{\mathrm{P}}^{\prime}-\boldsymbol{R}_{\mathrm{P}}\right] \boldsymbol{q}\right\} \\
& V_{\mathrm{tp}}=\frac{1}{2 \pi^{2}} \int \frac{\mathrm{d} \boldsymbol{q}}{q^{2}} \exp \left[\mathrm{i}\left(\beta \boldsymbol{r}_{\mathrm{P}}-\boldsymbol{r}_{\mathrm{P}}^{\prime}\right) \boldsymbol{q}\right] \\
& V_{\mathrm{Pt}}=-\frac{1}{2 \pi^{2}} Z_{\mathrm{P}} \int \frac{\mathrm{d} \boldsymbol{q}}{q^{2}} \exp \left\{\mathrm{i}\left[(1-\beta) \boldsymbol{r}_{\mathrm{P}}+\boldsymbol{r}_{\mathrm{P}}^{\prime}\right] \boldsymbol{q}\right\} .
\end{aligned}
$$

We first remark that the third term in (3.11) includes the factor

$$
\left\langle\varphi_{f}^{\mathrm{P}}\left|\exp \left[\mathrm{i}(1-\beta) \boldsymbol{r}_{\mathrm{P}} \cdot \boldsymbol{q}\right]\right| \varphi_{\mathrm{i}}^{\mathrm{P}}\right\rangle \sim \mathrm{O}\left(m / M_{\mathrm{P}}\right)
$$

since $\beta=1+\mathrm{O}\left(m / M_{\mathrm{P}}\right)$ and $\left\langle\varphi_{i}^{\mathrm{P}} \mid \varphi_{f}^{\mathrm{P}}\right\rangle=0$. Hence the term (3.13) can be neglected to within an error of the order $m / M_{\mathrm{P}}$. The remaining two terms in (3.11) correspond to the electron-electron and the target-nucleus-projectile-electron interaction. Transforming $\varphi_{f}^{\top}$ into momentum space, we obtain (Thumm 1985)

$$
\begin{aligned}
& T_{i f}^{(\mathrm{SPB})}=\frac{2}{(2 \pi)^{13 / 2}} \int \frac{\mathrm{d} \boldsymbol{q}}{q^{2}} \mathrm{~d} \boldsymbol{k} \mathrm{d} \boldsymbol{k}^{\prime} \mathrm{d} \boldsymbol{r}_{\mathrm{P}}^{\prime} \mathrm{d} \boldsymbol{R}_{\mathrm{P}} \varepsilon_{i f}^{\mathrm{P}}(-\beta \boldsymbol{q}) \exp \left(-\mathrm{i} \boldsymbol{K}_{f} \boldsymbol{R}_{\mathrm{T}}\right) \\
& \quad \times \tilde{\varphi}_{f}^{\mathrm{T}^{*}}\left(\boldsymbol{k}^{\prime}\right) \exp \left(-\mathrm{i} \boldsymbol{k}^{\prime} \cdot \boldsymbol{r}_{\mathrm{T}}\right)\left\{\exp \left(-\mathrm{i} \boldsymbol{q} \cdot \boldsymbol{r}_{\mathrm{P}}^{\prime}\right)-Z_{\mathrm{T}} \exp \left[-\mathrm{i}\left(1-\beta^{\prime}\right) \boldsymbol{q} \cdot \boldsymbol{r}_{\mathrm{P}}^{\prime}-\mathrm{i} \boldsymbol{q} \cdot \boldsymbol{R}_{\mathrm{P}}\right]\right\} \\
& \quad \times \exp \left[-\mathrm{i} \beta^{-1}\left(\boldsymbol{k}+\boldsymbol{K}_{i}\right) \boldsymbol{R}_{\mathrm{P}}\right] \Psi_{\boldsymbol{k}, \varepsilon}^{+}\left(\boldsymbol{r}_{\mathrm{P}}^{\prime}\right) \tilde{\varphi}_{i}^{\mathrm{T}}\left(\beta^{\prime-1} \boldsymbol{k}+\boldsymbol{v}\right) \\
&= \frac{1}{2 \pi^{2}} \int \frac{\mathrm{d} \boldsymbol{q}}{q^{2}} \mathrm{~d} \boldsymbol{k} \varepsilon_{i f}^{\mathrm{P}}(-\beta \boldsymbol{q}) \tilde{\Psi}_{k, \varepsilon}^{+}(\boldsymbol{K}+\boldsymbol{k}+\boldsymbol{q}) \tilde{\varphi}_{i}^{\mathrm{T}}\left(\beta^{-1} \boldsymbol{k}+\boldsymbol{v}\right) \\
& \times\left[\tilde{\varphi}_{f}^{\mathrm{T}^{*}}\left(-\alpha \boldsymbol{K}_{f}+\beta^{\prime-1} \boldsymbol{K}_{i}+\beta^{\prime-1} \boldsymbol{k}\right)-Z_{\mathrm{T}} \tilde{\varphi}_{f}^{\mathrm{T}^{*}}\left(-\alpha \boldsymbol{K}_{f}+\beta^{\prime-1} \boldsymbol{K}_{i}+\beta^{\prime-1} \boldsymbol{k}+\boldsymbol{q}\right)\right]
\end{aligned}
$$

where the projectile form factor

$$
\varepsilon_{i f}^{\mathrm{P}}(\boldsymbol{K})=\left\langle\boldsymbol{\varphi}_{f}^{\mathrm{P}}\left|\exp \left(-\mathrm{i} \boldsymbol{K} \cdot \boldsymbol{r}_{\mathrm{P}}\right)\right| \boldsymbol{\varphi}_{i}^{\mathrm{P}}\right\rangle
$$


and the momentum transfer vector

$$
\boldsymbol{K} \equiv \boldsymbol{K}_{i}-\boldsymbol{K}_{f}
$$

have been introduced.

For small momentum transfers $\boldsymbol{K}$, i.e. $\boldsymbol{K}_{f} \simeq \boldsymbol{K}_{i}=\boldsymbol{M} \boldsymbol{v},-\alpha \boldsymbol{K}_{f}+\beta^{\prime-1} \boldsymbol{K}_{i} \simeq \boldsymbol{K}+\boldsymbol{v}$ and within errors of the order $m / M(3.14)$ can be rewritten as

$$
\begin{aligned}
T_{i f}^{(\mathrm{SPB})}=\frac{1}{2 \pi^{2}} \int \frac{\mathrm{d} \boldsymbol{q}}{q^{2}} \mathrm{~d} \boldsymbol{k} \varepsilon_{i f}^{\mathrm{P}}(-\boldsymbol{q}) \tilde{\Psi}_{\boldsymbol{k}-v, \varepsilon}^{+}(\boldsymbol{K}+\boldsymbol{k}-\boldsymbol{v}+\boldsymbol{q}) \tilde{\varphi}_{i}^{\mathrm{T}}(\boldsymbol{k})\left[\tilde{\varphi}_{f}^{\mathrm{T}^{*}}(\boldsymbol{K}+\boldsymbol{k})\right. \\
\left.-Z_{\mathrm{T}} \tilde{\varphi}_{f}^{\mathrm{T}^{* *}}(\boldsymbol{K}+\boldsymbol{k}+\boldsymbol{q})\right] .
\end{aligned}
$$

To the order $(m / M)$ the expression (3.17) is exact. However the execution of the six-dimensional integral presents formidable numerical problems. In order to render this integral tractable and, more importantly, to illuminate the precise relationship to the first Born approximation we will make two approximations. The first is to put the distorted wave $\tilde{\Psi}_{k-v, \varepsilon}^{+}$on shell, i.e. to treat the target electron as if it were initially free. This involves the replacement of $\Psi_{k-v, \varepsilon}^{+}(\boldsymbol{r})$ by the on-shell wavefunction $\left(\boldsymbol{k}^{\prime} \equiv \boldsymbol{k}-\boldsymbol{v}\right)$

$$
\Psi_{\boldsymbol{k}^{\prime}}^{+}(\boldsymbol{r})=(2 \pi)^{-3 / 2} \exp \left(\mathrm{i} \boldsymbol{k}^{\prime} \cdot \boldsymbol{r}\right) G_{\boldsymbol{k}^{\prime}}(\boldsymbol{r})
$$

where we have defined

$$
G_{\boldsymbol{k}^{\prime}}(\boldsymbol{r})=N(\nu)_{1} F_{1}\left(-\mathrm{i} \nu, 1 ; \mathrm{i}\left(k^{\prime} \boldsymbol{r}-\boldsymbol{k}^{\prime} \cdot \boldsymbol{r}\right)\right)
$$

with

$$
N(\nu)=\exp (-\pi \nu / 2) \Gamma(1+\mathrm{i} \nu)
$$

and

$$
\nu=-\left(Z_{\mathrm{P}}-1\right) / k^{\prime}
$$

This on-shell approximation allows (3.17) to be replaced by

$$
\begin{aligned}
& T_{i f}^{(\mathrm{SPB})}=\frac{1}{2 \pi^{2}} \int \mathrm{d} \boldsymbol{q} q^{-2} \varepsilon_{i f}^{\mathrm{P}}(-\boldsymbol{q}) \int \mathrm{d} \boldsymbol{k} \tilde{\varphi}_{i}^{\mathrm{T}}(\boldsymbol{k}) \\
& (2 \pi)^{-3 / 2} \tilde{G}_{\boldsymbol{k}-v}(\boldsymbol{K}+\boldsymbol{q})\left[\tilde{\varphi}_{f}^{\mathrm{T}^{*}}(\boldsymbol{K}+\boldsymbol{k})-Z_{\mathrm{T}} \tilde{\boldsymbol{\varphi}}_{f}^{\mathrm{T}^{*}}(\boldsymbol{K}+\boldsymbol{k}+\boldsymbol{q})\right] .
\end{aligned}
$$

We note that the term $\tilde{G}$ is the only velocity-dependent part of $(3.22)$ and represents the velocity-dependent polarisation of the target wavefunction. The first Born approximation removes this velocity dependence by replacing $G_{k^{\prime}}(\boldsymbol{r})$ in (3.18) by unity, which gives $\tilde{G}_{k-v}(\boldsymbol{K}+\boldsymbol{q})=(2 \pi)^{3 / 2} \delta(\boldsymbol{K}+\boldsymbol{q})$ independent of $\boldsymbol{v}$ and $\boldsymbol{k}$. Then (3.22) reduces to

$$
T_{i f}^{(\mathrm{B} 1)}=\frac{1}{2 \pi^{2}} K^{-2} \varepsilon_{i f}^{\mathrm{p}}(\boldsymbol{K})\left(\varepsilon_{i f}^{\mathrm{T}}(\boldsymbol{K})-Z_{\mathrm{T}} \delta_{i f}\right)
$$

as given by Briggs and Taulbjerg (1978). To evaluate (3.22) further we make a peaking approximation whose validity rests upon the fact that $Z_{\mathrm{P}} \gg Z_{\mathrm{T}}$ and we restrict ourselves to velocities $v \gg Z_{\mathrm{T}}$. Then one recognises that $\tilde{\varphi}_{i}^{\mathrm{T}}(\boldsymbol{k})$ is strongly peaked near $k=0$ in momentum space and hence one can approximate $\tilde{G}_{k-v}$ by its value $\tilde{G}_{-v}$ taken at $k=0$. This allows the $\boldsymbol{k}$ integral in $(3.22)$ to be rewritten to give

$$
T_{i f}^{(\mathrm{SPB})}=\frac{1}{2 \pi^{2}} \int \mathrm{d} \boldsymbol{q} q^{-2} \varepsilon_{i f}^{\mathrm{P}}(-\boldsymbol{q}) \tilde{G}_{-v}(\boldsymbol{K}+\boldsymbol{q})\left[\varepsilon_{i f}^{\mathrm{T}}(\boldsymbol{K})-Z_{\mathrm{T}} \varepsilon_{i f}^{\mathrm{T}}(\boldsymbol{K}+\boldsymbol{q})\right] .
$$


The second step of the peaking approximation is to recognise that $\tilde{G}_{-v}(\boldsymbol{K}+\boldsymbol{q})$ is peaked near $\boldsymbol{K}+\boldsymbol{q}=0$ (in the first Born approximation it is a $\delta$ function) and to replace $\boldsymbol{q}$ by $-\boldsymbol{K}$ in the rest of the integrand, except the term $\varepsilon_{\text {if }}^{\mathrm{T}}(\boldsymbol{K}+\boldsymbol{q})$ which also has a peak at $\boldsymbol{K}=-\boldsymbol{q}$, when $f=i$. This approximation allows the $T$-matrix element to be written $T_{i f}^{(\mathrm{SPB})}=\frac{1}{2 \pi^{2}} K^{-2}\left[\varepsilon_{i f}^{\mathrm{P}}(\boldsymbol{K}) G_{-v}(0) \varepsilon_{i f}^{\mathrm{T}}(\boldsymbol{K})-Z_{\mathrm{T}} \varepsilon_{i f}^{\mathrm{P}}(\boldsymbol{K}) \int \mathrm{d} \boldsymbol{q} \tilde{G}_{-\boldsymbol{v}}(\boldsymbol{K}+\boldsymbol{q}) \varepsilon_{i f}^{\mathrm{T}}(\boldsymbol{K}+\boldsymbol{q})\right]$.

In fact the peaking in the first term, arising from the electron-electron interaction, can be relaxed somewhat in that only the factor $q^{-2}$ is withdrawn from the integral with the result that $\varepsilon_{i f}^{\mathrm{P}}(\boldsymbol{K}) G_{-\boldsymbol{v}}(0)$ is replaced by

$$
\bar{\varepsilon}_{i f}^{\mathrm{P}}(\boldsymbol{v}, \boldsymbol{K}) \equiv\left\langle\varphi_{f}^{\mathrm{P}}\left|G_{-\boldsymbol{v}}(\boldsymbol{r}) \exp (-\mathrm{i} \boldsymbol{K} \cdot \boldsymbol{r})\right| \varphi_{i}^{\mathrm{P}}\right\rangle .
$$

For subsequent calculation this more accurate form will be used. Finally we recognise that

$$
\int \mathrm{d} \boldsymbol{q} \tilde{G}_{-v}(\boldsymbol{K}+\boldsymbol{q}) \varepsilon_{i f}^{\mathrm{T}}(\boldsymbol{K}+\boldsymbol{q})=\left\langle\boldsymbol{\varphi}_{f}^{\mathrm{T}}\left|\boldsymbol{G}_{-\boldsymbol{v}}(\boldsymbol{r})\right| \boldsymbol{\varphi}_{i}^{\mathrm{T}}\right\rangle
$$

and is independent of $\boldsymbol{K}$. Hence the $T$-matrix element (3.24) is also approximated by the form

$$
T_{i f}^{\mathrm{SPB}}=\left(1 / 2 \pi^{2}\right) K^{-2}\left(\bar{\varepsilon}_{i f}^{\mathrm{P}}(\boldsymbol{v}, \boldsymbol{K}) \varepsilon_{i f}^{\mathrm{T}}(\boldsymbol{K})-Z_{\mathrm{T}} \varepsilon_{i f}^{\mathrm{P}}(\boldsymbol{K})\left\langle\boldsymbol{\varphi}_{f}^{\mathrm{T}}\left|G_{-\boldsymbol{v}}(\boldsymbol{r})\right| \varphi_{i}^{\mathrm{T}}\right\rangle\right) .
$$

This form illustrates clearly that when we put $G_{-v}(\boldsymbol{r}) \simeq 1$ we recover the first Born approximation, consistent with (3.18).

In most cases projectile excitation has been measured without specifying the final target state (Schiebel et al 1977, Tawara et al 1978, 1979, Terasawa et al 1983, Wohrer et al 1986). We therefore sum in closure approximation over the complete set of final target states. We write the total cross section for projectile excitation $\sigma_{i f}$ as a sum of the singly inelastic part $\sigma_{i f, 0}$, where the target remains in its ground state, and a doubly inelastic contribution $\Sigma_{f^{\prime} \neq 0} \sigma_{i f, f^{\prime}}$ which includes simultaneous excitation of target and projectile:

$$
\begin{aligned}
& \sigma_{i f}=\sigma_{i f, 0}+\sum_{f^{\prime} \neq 0} \sigma_{i f, f^{\prime}} \\
& \sigma_{i f, 0}=\frac{(2 \pi)^{5}}{v^{2}} \int_{K_{\text {min }}}^{K_{\text {max }}} \mathrm{d} K K\left|T_{i f, 0}\right|^{2}, K_{\min }=\frac{\Delta \varepsilon^{p}}{v} \\
& \sum_{f^{\prime} \neq 0} \sigma_{i f, f^{\prime}}=\frac{(2 \pi)^{5}}{v^{2}} \int_{\bar{K}}^{K_{\max }} \mathrm{d} K K \sum_{f^{\prime} \neq 0}\left|T_{i f}\right|^{2} \quad \bar{K}=\left(\Delta \varepsilon^{\mathrm{P}}+\Delta \bar{\varepsilon}^{\mathrm{T}}\right) / v .
\end{aligned}
$$

Since the most important contribution to the sum in $(3.29 \mathrm{c})$ is due to low lying excited target states, the averaged target excitation energy $\Delta \bar{\varepsilon}^{-\mathrm{T}}$ is chosen as the $1 \mathrm{~s} \rightarrow 2 \mathrm{p}$ excitation energy $\Delta \varepsilon_{1 \mathrm{~s}, 2 \mathrm{p}}^{\mathrm{T}}$. In general (3.29) depends strongly on $K_{\min }$ and $\bar{K}$ and the doubly inelastic cross section $(3.29 \mathrm{c})$ is sensitive to the chosen value of $\Delta \bar{\varepsilon}^{\mathrm{T}}$. However, for $1 \mathrm{~s} \rightarrow f$ excitation of heavy projectile ions colliding with light targets, $\bar{K}$ is principally determined by $\Delta \varepsilon_{1 \mathrm{~s}, f}^{\mathrm{P}} \gg \Delta \bar{\varepsilon}^{\mathrm{T}}$, such that the uncertainty in the choice of $\Delta \bar{\varepsilon}^{\mathrm{T}}$ does not influence the final results significantly. In other words, the closure over final target states is nearly exact.

Using the completeness relation

$$
\sum_{f^{\prime} \neq 0}\left|\varphi_{f^{\prime}}^{\mathrm{T}}\right\rangle\left\langle\varphi_{f^{\prime}}^{\mathrm{T}}|=1-| \varphi_{0}^{\mathrm{T}}\right\rangle\left\langle\varphi_{0}^{\mathrm{T}}\right|
$$


the sum in $(3.29 c)$ disappears and $(3.29 b, c)$ can now be written in the final form

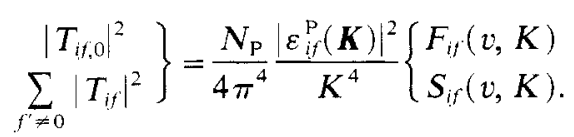

$F_{i f}$ and $S_{i f}$ are generalisations of the coherent and incoherent scattering functions (Kim and Inokuti 1968). They are given in terms of form factors and overlap integrals by

$$
\begin{aligned}
& F^{\mathrm{B} 1}(K)=\left|N_{\mathrm{T}} \varepsilon_{00}^{\mathrm{T}}(\boldsymbol{K})-Z_{\mathrm{T}}\right|^{2} \\
& F_{i f}^{\mathrm{SPB}}(v, K)=|N(\nu)|^{2}\left|N_{\mathrm{T}} g_{i f}(v, K) \varepsilon_{00}^{\mathrm{T}}(\boldsymbol{K})-Z_{\mathrm{T}} f(v)\right|^{2}
\end{aligned}
$$

and (we now suppress the arguments on the right-hand side)

$$
\begin{gathered}
S^{\mathrm{B} 1}(K)=N_{\mathrm{T}}\left(1-\left|\varepsilon_{00}^{\mathrm{T}}\right|^{2}\right) \\
S_{i f}^{\mathrm{SPB}}(v, K)=\mid N^{2}\left\{N_{\mathrm{T}}\left|g_{i f}\right|^{2}\left(1-\left|\varepsilon_{00}^{\mathrm{T}}\right|^{2}\right)+Z_{\mathrm{T}}^{2}\left(r-|f|^{2}\right)-2 N_{\mathrm{T}} Z_{\mathrm{T}} \operatorname{Re}\left[g_{i f}^{*}(s-f)\right] \varepsilon_{00}^{\mathrm{T}}\right\}
\end{gathered}
$$

for the first Born and SPB approximations, respectively. In equations $(3.32 b)$ and $(3.33 b)$ we have defined

$$
\begin{array}{cc}
f(v) \equiv \frac{\left\langle\varphi_{0}^{\mathrm{T}}\left|G_{-v}\right| \varphi_{0}^{\mathrm{T}}\right\rangle}{N(\nu)} & g_{i f}(v, K) \equiv \frac{\bar{\varepsilon}_{i f}^{\mathrm{P}}(\boldsymbol{v}, \boldsymbol{K})}{N(\nu) \varepsilon_{i f}^{\mathrm{P}}(\boldsymbol{K})} \\
r(v) \equiv \frac{\left\|G_{-\boldsymbol{v}} \varphi_{0}^{\mathrm{T}}\right\|^{2}}{|N(\nu)|^{2}} & s(v, K) \equiv \frac{\bar{\varepsilon}_{00}^{\mathrm{T}}(\boldsymbol{v}, \boldsymbol{K})}{N(\nu) \varepsilon_{00}^{\mathrm{T}}(\boldsymbol{K})}
\end{array}
$$

and

$$
\bar{\varepsilon}_{00}^{\mathrm{T}}(\boldsymbol{v}, \boldsymbol{K}) \equiv\left\langle\boldsymbol{\varphi}_{0}^{\mathrm{T}}\left|G_{-\boldsymbol{v}} \exp (-\mathrm{i} \boldsymbol{K} \cdot \boldsymbol{r})\right| \boldsymbol{\varphi}_{0}^{\mathrm{T}}\right\rangle
$$

In contrast to the SPB scattering functions the scattering functions in the Born approximation are $v$ independent. Another new feature in comparison with the first Born approximation is that the inelastic scattering function $(3.33 b)$ depends on the targetnucleus-projectile-electron interaction, which follows from the fact that the distorted initial target state is not orthogonal to final excited states of the target. The functions (3.34) are defined in such a way that their deviation from 1 indicates the strength of the polarisation. If they are replaced by 1 the scattering functions reduce to their first Born expressions. The factors $N_{\mathrm{T}}$ and $N_{\mathrm{P}}$ are equal to 1 or 2 according to whether the appropriate $\mathrm{K}$ shell is singly or doubly occupied initially.

\section{Results}

In view of the approximate evaluation of the SPB $T$-matrix element in (3.17), leading to the tractable form (3.28), no great accuracy of our calculations is claimed although the correct qualitative features of a more exact evaluation are retained. Rather we emphasise these qualitative features with the main aim of indicating that for collisions involving highly charged ions the first Born approximation is never valid at nonrelativistic collision velocities. As examples we consider the excitation $1 \mathrm{~s}^{2}{ }^{1} \mathrm{~S} \rightarrow 1 \mathrm{~s} 2 \mathrm{p}{ }^{1} \mathrm{P}$ of $\mathrm{F}^{7+}$ and $\mathrm{Fe}^{24+}$ ions in collisions with neutral helium. In each case we consider the features of the first Born and SPB approximations in the appropriate velocity regions. 

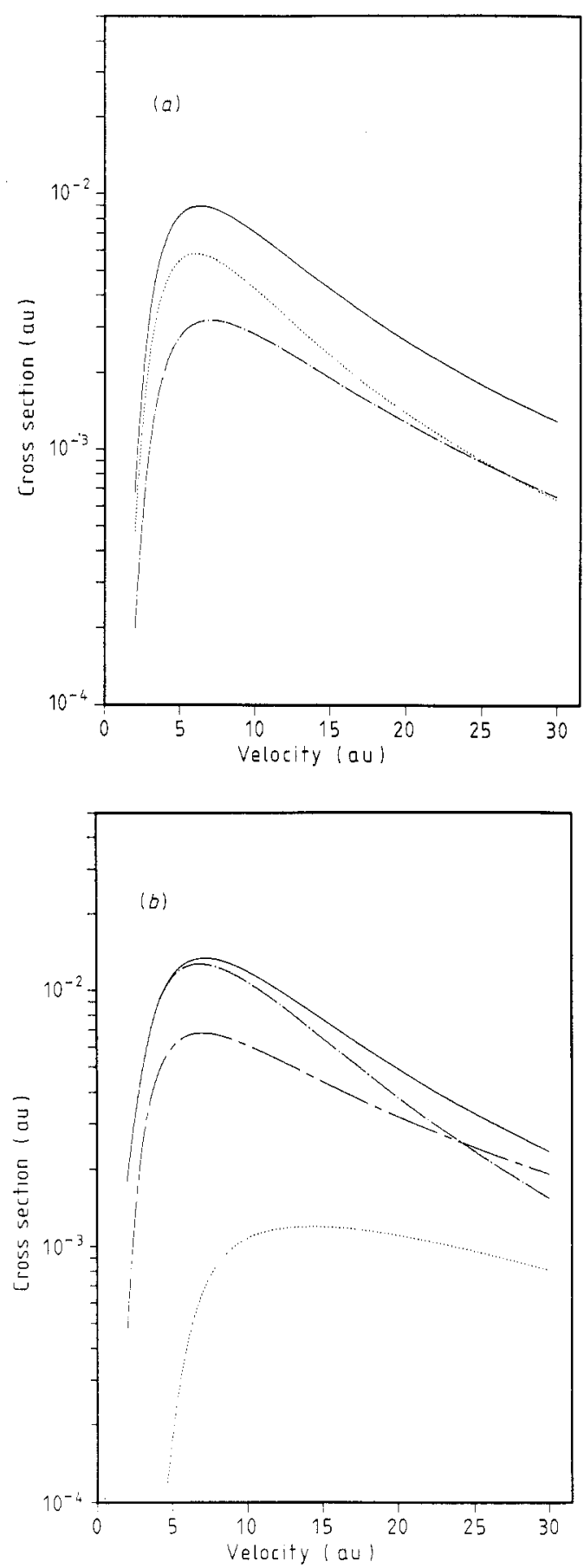

Figure 2. Total cross section for $1 s^{2}{ }^{1} \mathrm{~S} \rightarrow 1 \mathrm{~s} 2 \mathrm{p}{ }^{1} \mathrm{P}$ excitation of $\mathrm{F}^{7+}$ colliding with neutral He. The individual singly $(\cdots)$ and doubly $(-\cdot-)$ inelastic contributions are also shown. (a) First Born approximation. (b) SPB approximation. The first Born cross section due to excitation by the target nucleus alone is also shown in $(b)$ for comparison $(--\infty)$. 

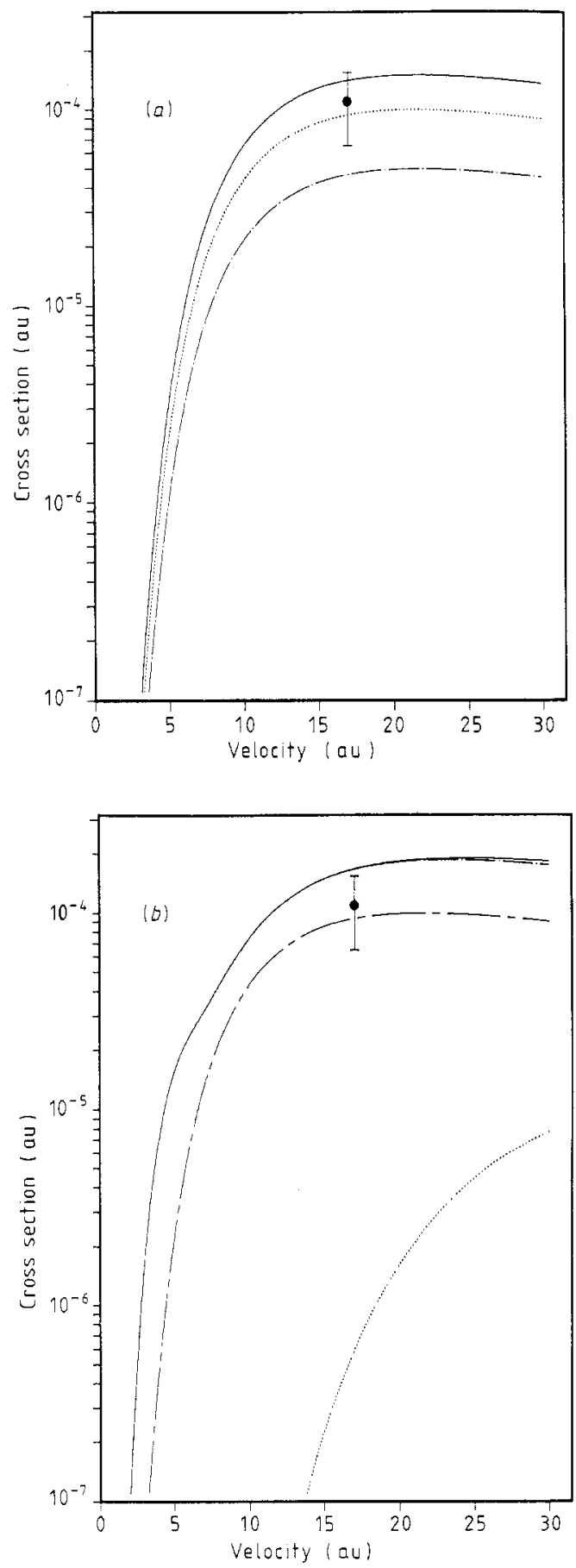

Figure 3. As figure 2 but for $\mathrm{Fe}^{24 t}$ projectiles. The experimental value is taken from Wohrer et al (1986). 
The wavefunctions have been taken to be of effective hydrogenic form, with full screening for the final and no screening for the initial projectile state and the Slater charge for the target state. The excitation energies are taken from experiment.

The total cross section for excitation of $\mathrm{F}^{7+}$ ions is shown in figure 2 , with the separate contributions of singly inelastic and doubly inelastic collisions indicated. At low collision velocities high momentum transfer collisions dominate $\left(K_{\min } \rightarrow \infty\right.$ as $v \rightarrow 0$ in (3.29b)) corresponding to very close collisions. Under these circumstances the target nucleus and electrons scatter essentially independently and hence, in the Born approximation, the relative contribution of singly inelastic (unscreened nuclear charge $Z_{\mathrm{p}}$ ) to doubly inelastic (two free electrons of charge unity) is approximately $Z_{\mathrm{P}}^{2} / 2=2$ in the case of helium. This accounts well for the relative magnitudes of the two contributions at and below the peak in the cross sections as shown in figure 2 . In this velocity region the singly inelastic contribution is also close to the first Born result for a bare helium nucleus. This is because the screening by the ground-state helium electrons plays no role for such short-distance collisions. By contrast at higher collision velocities, $v \gg Z_{\mathrm{P}}$, corresponding to smaller momentum transfers and larger distance collisions, the screening effect becomes dominant (the helium atom behaves as a neutral particle) and the excitation cross section decreases rapidly below that due to a bare nucleus and also below that due to the target electrons alone (the doubly inelastic contribution). The SPB result (figure $2 b$ ), incorporating exchange distortion of the target, shows a strong deviation from the first Born result. The major change is a drastic reduction in the singly inelastic cross section at low velocity. This is quite simply due to the large polarising effect of the projectile nuclear charge on the helium ground state which makes it highly unlikely that in a close collision the helium atom will penetrate undistorted to short distances. In turn this implies that at low velocities the doubly inelastic contribution completely dominates the total cross section. However, as the collision velocity increases the relative contribution of the singly inelastic cross section also increases due to the reduced polarisation effect. The doubly inelastic cross section at low velocity is far greater than that in the first Born approximation. In the latter this contribution arises solely from the electron-electron interaction. The SPB matrix element does not factor in this way and the doubly inelastic contribution is due to a coherent sum of the electron and nuclear fields. This further accounts for the fact that this contribution alone at low velocities exceeds that from the first Born approximation for a bare nucleus.

All these qualitative features are exaggerated further by an increase in projectile nuclear charge, as shown in figure 3 for the case of collisions of $\mathrm{Fe}^{24+}$ ions with helium. In this case the ratio of singly to doubly inelastic collisions is almost exactly $2: 1$ in the non-relativistic velocity region in the first Born approximation (figure $3(a)$ ).

The singly inelastic contribution is in this case identical with that due to the target nucleus alone, indicating that for these short distances of approach the screening by target electrons plays no role. Interestingly the total cross section agrees with the one available data point. That this agreement is fortuitous is demonstrated in the SPB approximation (figure $3(b)$ ) in which the singly inelastic contribution contributes a negligible amount to the total cross section. This is to be expected since a helium atom in its ground state cannot penetrate to the $\mathrm{K}$ shell of $\mathrm{Fe}$ without itself being severely distorted (i.e. excited, ionised or captured). The doubly inelastic cross section sums over these processes. Again the doubly inelastic cross section, arising from a coherent superposition of the exciting fields of target electrons and nucleus, lies above that of the nucleus alone and is in good agreement with the experimental measurement. 


\section{Conclusions}

We have re-examined the theory of excitation in ion-atom collisions in the situation where one of the nuclear fields is so strong that the condition $Z / v \ll 1$ (where $Z$ is the nuclear charge and $v$ the collision velocity) cannot be met at non-relativistic velocities. This invalidates the first Born approximation. For the particular case of K-shell excitation of a highly charged projectile, the strong polarising effect of the projectile on a neutral target has been taken into account by a Faddeev expansion of the full Green operator for the ion-atom system. This leads to a corrected form of the first Born approximation in which the expressions for singly and doubly inelastic scattering cross sections contain form factors which differ from their first Born forms by the inclusion of terms expressing the polarisation of the target electrons. An evaluation of these expressions results in a strongly reduced contribution of singly inelastic scattering. The total cross section is dominated by doubly inelastic events in which the projectile electron is excited by the coherent operation of the target electrons and nucleus.

\section{References}

Bates D R and Griffing G W 1953 Proc. Phys. Soc. A 66 961-71 1954 Proc. Phys. Soc. A $67663-8$ 1955 Proc. Phys. Soc. A 68 90-5

Brendlé B, Gayet R, Rozet J P and Wohrer K 1985 Phys. Rev, Lett. 54 2007-10

Briggs J S 1976 Rep. Prog. Phys. 39 217-89

Briggs J S and Taulbjerg K 1978 Topics in Current Physics vol 5, ed I A Sellin (New York: Springer) pp 105-53

Coleman J P 1969 Case Studies in Atomic Collision Physics vol 1 (Amsterdam: North-Holland) p 101

Gillespie G H, Kim Y-K and Cheng K T 1978 Phys. Rev. A 17 1284-95

Hartley H M and Walters H R J 1987 J. Phys. B: At. Mol. Phys. 20 3811-31

Kim Y-K and Inokuti M 1968 Phys. Rev. $16539-43$

McDowell M R C 1961 Proc. R. Soc. A 264277

Macek J and Alston S 1982 Phys. Rev. A 26250

Schiebel U, Doyle B L, Macdonald J R and Ellsworth L D 1977 Phys. Rev. A 16 1089-92

Tawara H, Richard P, Jamison K A and Gray T J 1978 J. Phys. B: At. Mol. Phys. 11 L615-20

Tawara H, Richard P, Jamison K A, Gray T J, Newcomb J and Schiedekamp C 1979 Phys. Rev. A 19 1960-70

Terasawa M, Gray T J, Hagmann S, Hall J, Newcomb J, Pepmiller P and Richard P 1983 Phys. Rev. A 27 $2868-75$

Thumm U 1985 Diplomarbeit Universität Freiburg

Wohrer K, Chetioui A, Rozet J P, Jolly A, Fernandez F, Stephan C, Brendlé B and Gayet R 1986 J. Phys. B: At. Mol. Phys. 19 1997-2006 